Samia Hurst est médecin, professeure de bioéthique et depuis peu membre de la rédaction du Bulletin des médecins suisses, ce dont je me réjouis particulièrement. Elle présente ici le Programme national de recherche «Fin de vie» (PNR 67) du Fonds national suisse de la recherche scientifique, qui a pour objectif d'acquérir plus de connaissances sur les décès, pour en particulier mieux évaluer les soins médicaux donnés, et mieux comprendre les évolutions sociales, et tenter de rendre plus humaine cette dernière phase de la vie. Le PNR 67 s'adresse en particulier aux groupes professionnels impliqués.

Dr Jürg Schlup, président de la FMH

\title{
Fin de vie: la réalité d'abord
}

Comment meurt-on en Suisse? Chacun d'entre nous a sans doute des craintes et des souhaits. Chacun d'entre nous a aussi des expériences personnelles. Songez à un patient que vous avez accompagné en fin de vie: ce sera sans doute à une situation qui a marqué, peut-être profondément, votre pratique ultérieure.

Lorsqu'il s'agit de discuter de comment accompagner au mieux ceux qui partent, ces expériences personnelles colorent nos avis. Nous pensons à ce qui s'est passé - mieux ou moins bien - dans les cas que nous connaissons. C'est légitime, mais notre expérience personnelle a des limites. Nous ne voyons pas tous les cas possibles, mais ceux que nous connaissons. Songez aux discussions publiques, vous mesurerez combien certaines sont éloignées de votre expérience.

Comment devrions-nous pouvoir mourir en Suisse? Autant que possible, sans souffrir. Mais dans combien de cas le soulagement de la souffrance est-il disponible, efficace? Devrions-nous pouvoir mourir sans technologie médicale? Déjà nous ne serons plus tous d'accord. Une mort plus ou moins «bonne» l'est-elle dans la mesure où elle est plus ou moins «naturelle», liée au terme spontané de la vie? Ou bien l'est-elle dans la mesure où elle reflète plus ou moins fidèlement les choix de vie de la personne qui meurt, y compris peut-être celui d'avoir recours le plus longtemps possible à tous les moyens de la médecine de pointe? Peut-être faudraitil que nous puissions chacun déterminer, autant que possible, ce que représente pour nous une mort meilleure qu'une autre [1]? Quelle est cependant l'implication réelle des patients dans les choix qui vont déterminer leur fin de vie? Dans les discussions sur comment accompagner au mieux les personnes qui partent, une série de questions factuelles sont essentielles.

Répondre à ces questions forme le socle d'une discussion saine sur les moyens, légaux et matériels, à mettre au service d'une mort qui puisse être la meilleure possible. L'étude sur les «Décisions médicales relatives à la fin de vie», financée par le FNS dans le cadre du PNR 67 et décrite dans ce numéro, est donc bienvenue. Etudier comment décèdent les personnes en se basant sur les témoignages des médecins ayant signé leurs certificats de décès, c'est à présent le gold standard international. Dans un nombre croissant de pays, ces études ont permis de recentrer le débat sur plus de réalité. Les décisions en fin de vie sont importantes: dans $2 / 3$ des cas, la mort est attendue et précédée d'une forme ou d'une autre de décision, en général une décision de limiter les moyens technologiques de la mé- decine de pointe [2]. Pour prendre un autre exemple, on a pu montrer pour la première fois en Belgique que légaliser la mort volontaire ne conduisait pas à plus de décès, mais à moins de silence [3]. On y a aussi constaté la progression des soins palliatifs après la légalisation de l'euthanasie [4].

Faire de bons choix sociétaux, donner les moyens d'une mort décente, voilà qui doit se fonder dans la réalité. Lorsque cette réalité est difficile à percevoir, des décalages se dessinent. Ces décalages ne sont pas inévitables, mais le prix pour les diminuer est que nous soyons prêts à décrire, clairement et réalistement, ce qui s'est réellement passé dans un

\section{Faire de bons choix sociétaux, donner les moyens d'une mort décente, voilà qui doit se fonder dans la réalité.}

certain nombre de cas où des médecins suisses auront accompagné leur patient jusqu'au bout. Comment meurt-on en Suisse? Ces données seront importantes. C'est finalement ce qui nous angoisse, ce comment. Plus que le fait même de mourir, semble-t-il. Cela mérite que nous y portions notre attention, aussi réticente qu'elle soit devant un tel sujet.

\section{Prof. Dr Samia Hurst, Institut d'éthique biomédicale,} Faculté de médecine, Genève

\section{Références}

1 Hurst SA, Mauron A. The ethics of palliative care and euthanasia: exploring common values. Palliat Med. 2006;20(2):107-12.

2 Van der Heide A, Deliens L, Faisst K, Nilstun T, Norup M, Paci E et al. End-of-life decision-making in six European countries: descriptive study. Lancet. 2003;362(9381):345-50.

3 Chambaere K, Bilsen J, Cohen J, Onwuteaka-Philipsen BD, Mortier F, Deliens L. Trends in medical end-of-life decision making in Flanders, Belgium 1998-2001-2007. Med Decis Making. 2011;31(3):500-10.

4 Pardon K, Chambaere K, Pasman HR, Deschepper R, Rietjens J, Deliens L. Trends in end-of-life decision making in patients with and without cancer. J Clin Oncol. 2013;31(11):1450-7. 